\title{
The role of histone deacetylase 6 (HDAC6) in neurodegeneration
}

\author{
This article was published in the following Dove Press journal: \\ Research and Reports in Biology \\ 2 September 2014 \\ Number of times this article has been viewed
}

Lawrence Van Helleputte ${ }^{1,2}$

Veronick Benoy ${ }^{1,2}$

Ludo Van Den Bosch ${ }^{1,2}$

'KU Leuven - University of Leuven, Department of Neurosciences,

Experimental Neurology and Leuven Research Institute for Neuroscience and Disease (LIND), Leuven, Belgium, ${ }^{2} \mathrm{VIB}$, Vesalius Research Center, Laboratory of Neurobiology, Leuven, Belgium
Correspondence: Ludo Van Den Bosch Laboratory of Neurobiology, Campus Gasthuisberg O\&N4, PB 912, Herestraat 49, B-3000 Leuven, Belgium

Tel +3216330681

Fax +32 I6 372534

Email ludo.vandenbosch@vib-kuleuven.be
Abstract: HDAC6 is an enzyme that regulates a variety of biological pathways in dividing cells, but also in post-mitotic neurons. In these cells, different cellular functions and survival are dependent on HDAC6-mediated processes such as intracellular trafficking, antioxidation, chaperone-mediated stress responses, and protein degradation. As a consequence, the interest in HDAC6 as a potential target to treat several neurodegenerative disorders has grown significantly over the last decade. This review summarizes the current knowledge on the interaction partners and functions of HDAC6 as well as the most important arguments for its involvement in several neurodegenerative diseases. As many of these disorders are hallmarked by alterations in HDAC6-mediated pathways, it is hypothesized that HDAC6 could play a pivotal role in the pathophysiology of neurodegeneration. HDAC6-dependent deacetylation of its substrates could result in neurotoxicity, while the ubiquitin-dependent functions of HDAC6 could be essential for neuroprotection. Therefore, targeting the deacetylating activity of HDAC6, while leaving its other functions unhampered, might be an interesting strategy to treat neurodegenerative disorders.

Keywords: HDAC6, (de)acetylation, neurodegeneration, axonal transport, autophagy, aggresome

\section{Introduction}

Histone deacetylases (HDACs) are enzymes that play an important role in transcriptional regulation by epigenetic modification of histones. ${ }^{1}$ Furthermore, some members of the HDAC family influence a variety of cellular processes through the modulation of non-histone substrates such as $\alpha$-tubulin, ubiquitin, HSP90, cortactin, peroxiredoxins, and several transcription factors. ${ }^{2-7}$

The HDAC family consists of 18 members, subdivided into four groups. According to their sequence homology to the yeast Rpd3 protein, HDAC1, 2, 3, and 8 are categorized as class I HDACs. These enzymes are ubiquitously expressed and localized in the nucleus, where they can influence transcription of genes. ${ }^{8}$ Class II HDACs, homologs of the yeast Hda1, show tissue-specific expression and are able to shuttle between the cytoplasm and the nucleus. They are further subdivided into class IIa (HDAC4, 5, 7, and 9) and IIb (HDAC6 and 10). Finally, HDAC11 is the only member of the class IV HDACs with similarities to the catalytic domains of both class I and II deacetylases. ${ }^{9}$ Class I, II, and IV HDACs are also referred to as the conventional HDACs and require $\mathrm{Zn}^{2+}$ as a cofactor for their deacetylating activity. ${ }^{10}$ The nicotinamide adenine dinucleotide-dependent sirtuins 1-7 are another class of HDAC enzymes (class III) and are homologs of Sir2 in yeast. ${ }^{11}$ 
Over the last decade, HDACs have been extensively studied for their role in cancer. Through histone deacetylation, the transcriptional regulation of tumor suppressor genes or oncogenes can be altered to influence the progression of cancer. ${ }^{12}$ As a consequence, HDAC inhibitors are a relatively new class of anticancer agents that induce cell cycle arrest and apoptosis in cancer cells and can reduce tumor growth. ${ }^{13}$ Although there are multiple clinical trials ongoing with broad spectrum HDAC inhibitors, only a handful of classor isoform-specific inhibitors are currently being tested in cancer-related studies. In general, HDAC inhibitors are well tolerated but some patients encountered adverse side effects such as nausea, thrombocytopenia, and fatigue. ${ }^{14}$ It is anticipated that isoform-specific inhibitors could have an improved efficacy and will be better tolerated during chronic treatment. From that perspective, the functions of specific subtypes of HDACs have been intensively studied and selective HDAC inhibitors are being developed. ${ }^{15}$

In particular, the interest in HDAC6 has grown over the past few years. It possesses some unique structural characteristics compared to the other HDAC isoforms. HDAC6 has two functional N-terminal catalytic deacetylating domains, a C-terminal zinc finger ubiquitin-binding domain (ZnF-UBP) and a specific tetradecapeptide repeat domain (SE14) that, together with two leucine-rich nuclear export sequences, promotes its cytosolic retention. ${ }^{16}$ These unique features allow HDAC6 to interact with non-histone substrates of which $\alpha$-tubulin, poly-ubiquitinated proteins, and HSP90 are the most studied ones. ${ }^{2,3,6}$ As such, HDAC6 can influence a wide range of cellular processes including cell growth, migration, and survival, but also protein degradation and intracellular trafficking. These latter processes are of pivotal importance for post-mitotic cells including neurons, which rely on efficient protein degradation and axonal transport for normal functioning. As a consequence, HDAC6 has emerged as a potential target for the treatment of different types of cancer as well as several neurodegenerative disorders, including Alzheimer's disease (AD), Parkinson's disease (PD), Huntington's disease (HD), and amyotrophic lateral sclerosis (ALS). This review will focus on the cellular functions of HDAC6 and its implications as a therapeutic target in different neurodegenerative diseases.

\section{The molecular functions of HDAC6 Regulation of microtubule dynamics by deacetylation of $\alpha$-tubulin}

Microtubules are cellular structures composed of $\alpha$ - and $\beta$-tubulin heterodimers that polymerize and depolymerize in a dynamic manner. Post-translational modifications of $\alpha$-tubulin control these dynamics ${ }^{17,18}$ and influence cell division, cell-cell interactions, cell migration, and intracellular trafficking. ${ }^{19}$ The discovery of $\alpha$-tubulin as a substrate for HDAC6 improved the insights in microtubule dynamics and its biological significance. HDAC6 tightly regulates deacetylation of $\alpha$-tubulin, which correlates to microtubule dynamics and proper functioning. ${ }^{20-22}$ Overexpression of HDAC6 completely deacetylates microtubules, while suppression of HDAC6 function, either by pharmacological inhibition or by small interfering RNA, elevates the levels of acetylated $\alpha$-tubulin, both in vitro and in vivo. ${ }^{20,22}$

Dynamic microtubules do not only orchestrate cell division, cell differentiation, and motility in dividing cells, ${ }^{23}$ but they are also of vital importance in post-mitotic neurons. ${ }^{24}$ These highly polarized cells require axonal transport of cargos from the cell body to the synapse and vice versa for normal cell function, neuronal plasticity, and survival. For this, neurons rely on the microtubule network, which functions as a track for molecular motors such as the kinesin and dynein superfamilies. ${ }^{25,26}$ Acetylation of $\alpha$-tubulin improves the interaction between the motor protein kinesin-1 and microtubules and promotes axonal trafficking. ${ }^{27} \mathrm{HDAC} 6$ inhibition also improves the binding of kinesin- 1 to $\alpha$-tubulin ${ }^{28}$ and HDAC6 can directly bind to p150 glued - a component of the dynein/dynactin motor complex. ${ }^{29}$ Indeed, knockdown of HDAC6 increases $\alpha$-tubulin acetylation, the recruitment of kinesin-1 and dynein to microtubules, and enhances mitochondrial transport along the axon in cultured neurons. ${ }^{27,28,30}$ In addition, other cellular pathways influence mitochondrial transport by regulating HDAC6 activity. For example, inhibition of the GSK-3 $\beta$ signaling pathway decreases HDAC6 activity, which in turn improves intracellular trafficking in hippocampal neurons. ${ }^{31}$ Taken together, HDAC6 can influence axonal transport, either directly by deacetylating $\alpha$-tubulin or indirectly by binding to motor proteins. For these reasons, HDAC6-mediated deacetylation is thought to be an important regulator of axonal transport in neurons.

\section{Regulation of the actin cytoskeleton by deacetylating cortactin}

In addition to its role in microtubule-dependent processes, HDAC6 can also influence remodeling of the actin cytoskeleton through deacetylation of cortactin. ${ }^{4}$ After stimulation with growth factors, cortactin translocates to the membrane ruffles where it can bind $\mathrm{F}$-actin to promote cell migration. ${ }^{4}$ HDAC6 is also enriched in these membrane ruffles where it deacetylates cortactin. Overexpression of HDAC6 improves 
the binding capacity of cortactin to F-actin and increases actin polymerization in vivo, whereas HDAC6 knockdown reduces cell motility. ${ }^{4}$

\section{Regulation of the antioxidant reactivity through peroxiredoxins}

Peroxiredoxins are antioxidants whose main function is to reduce $\mathrm{H}_{2} \mathrm{O}_{2}$ concentrations in cells. ${ }^{32}$ PRXI and PRXII have recently been added to the list of HDAC6 substrates. Under conditions of extreme oxidative stress, intracellular $\mathrm{H}_{2} \mathrm{O}_{2}$ concentrations reach a critical threshold after which PRXI and PRXII oligomerize to high-molecular-weight complexes. After this transformation, peroxiredoxins lose their peroxidase activity and function as molecular chaperones instead of antioxidants. ${ }^{33}$ Interestingly, high levels of acetylation improved the reducing activity as well as the resistance to oligomerization and overoxidation of PRXI and PRXII in vitro. ${ }^{7}$ Inhibition of the deacetylating function of HDAC6 restores the peroxidase activity of PRXI and PRXII during periods of high oxidative stress. ${ }^{7}$ In conclusion, these results indicate that inhibition of HDAC6 can result in a beneficial increase of antioxidant reactivity of peroxiredoxins.

\section{Regulation of the anti- inflammatory response}

In health, the immune system is in perfect homeostasis. Upon infection or disease, the proinflammatory response is enhanced to eliminate toxic insults. Once the pathogen is cleared, anti-inflammatory signals temper that response in order to preserve the surrounding tissue. However, aberrant activation of the immune system can result in chronic inflammation, which is often observed in neurodegenerative disorders such as AD, PD, and ALS. ${ }^{34,35}$ Key regulators of inflammation are Foxp3-expressing regulatory T-cells. These cells reduce the expression of proinflammatory cytokines and suppress the activation of effector T-lymphocytes, thus tempering immune reactions that are detrimental for the host and restoring immunological homeostasis. ${ }^{36}$ Recently, HDACs have been implicated as modulators of the immune response by promoting the expression of proinflammatory genes and HDAC inhibitors are emerging as anti-inflammatory agents. ${ }^{37}$ Although it was suggested that the immunosuppressive effects were due to histone modification, HDAC6 has been shown to regulate the Foxp $3^{+}$regulatory T-cells through HSP90 modulation..$^{38}$ Genetic deletion or pharmacological inhibition of HDAC6 increased the suppressive activity of regulatory T-cells in models for autoimmunity and prevented allograft rejection..$^{38}$ Since improving the regulatory T-cell function limits neuroinflammation, ${ }^{39,40}$ HDAC6 inhibitors could reduce chronic inflammation in autoimmune disorders and neurodegeneration.

\section{Regulation of the chaperoning activity of HSP90}

In 2005, HSP90 was identified as a substrate of HDAC6. ${ }^{3}$ This molecular chaperone fulfills a pivotal role in diverse signaling pathways. It facilitates the maturation and assembly of transcription factors and other client proteins by improving their stability as well as their activity. ${ }^{41}$ It was shown that HDAC6 regulates the chaperone activity of HSP90 by reversible deacetylation. ${ }^{3}$ Furthermore, HSP90 binds to and regulates the activity of HSF1, a key enzyme in the response to cellular stress. ${ }^{42}$ As discussed in the section on autophagy, HDAC6 was identified as a component of a repressive complex where it senses the presence of ubiquitinated aggregates and triggers the dissociation of this complex, activating a protective response to cytotoxic insults. ${ }^{43}$ As HDAC6 regulates HSP90 activity, it most likely is a modifier of prooncogenic pathways mediated by HSP90. Indeed, both HSP90 inhibitors ${ }^{44}$ and HDAC6 inhibitors ${ }^{45}$ are useful as anticancer therapies by destabilizing the HDAC6-HSP90 interactions. Furthermore, a recent study reported that both HDAC6 silencing and HSP90 knockdown in preclinical models of prostate cancer result in a downregulation of the androgen receptor and Akt expression, counteracting the resistance of tumor cells to hormone therapy. ${ }^{46}$ These results indicate that HDAC6 potentially modulates the function of other proteins by regulating HSP90 activity. In contrast to this, a recent study suggests that the positive effects of HDAC6 inhibition in prostate cancer cells correlates with cortactin-dependent cell motility rather than HSP90 regulation. ${ }^{47}$

\section{Regulation of cellular pathways for protein-induced stress responses}

Cells are able to cope with stress induced by abnormal proteins through a variety of pathways such as the ubiquitinproteasome system and autophagy (Figure 1).

\section{The ubiquitin-proteasome system}

The ubiquitin-proteasome system is the main cellular mechanism for protein degradation. Misfolded or damaged proteins receive a poly-ubiquitin tag, after which they are directed to the proteasome for subsequent degradation. ${ }^{48}$ However, when the protein load is too high, the proteasome can become saturated and cytotoxic aggregates will accumulate. In such cases, often observed in neurodegenerative disorders, ${ }^{49}$ cells must 


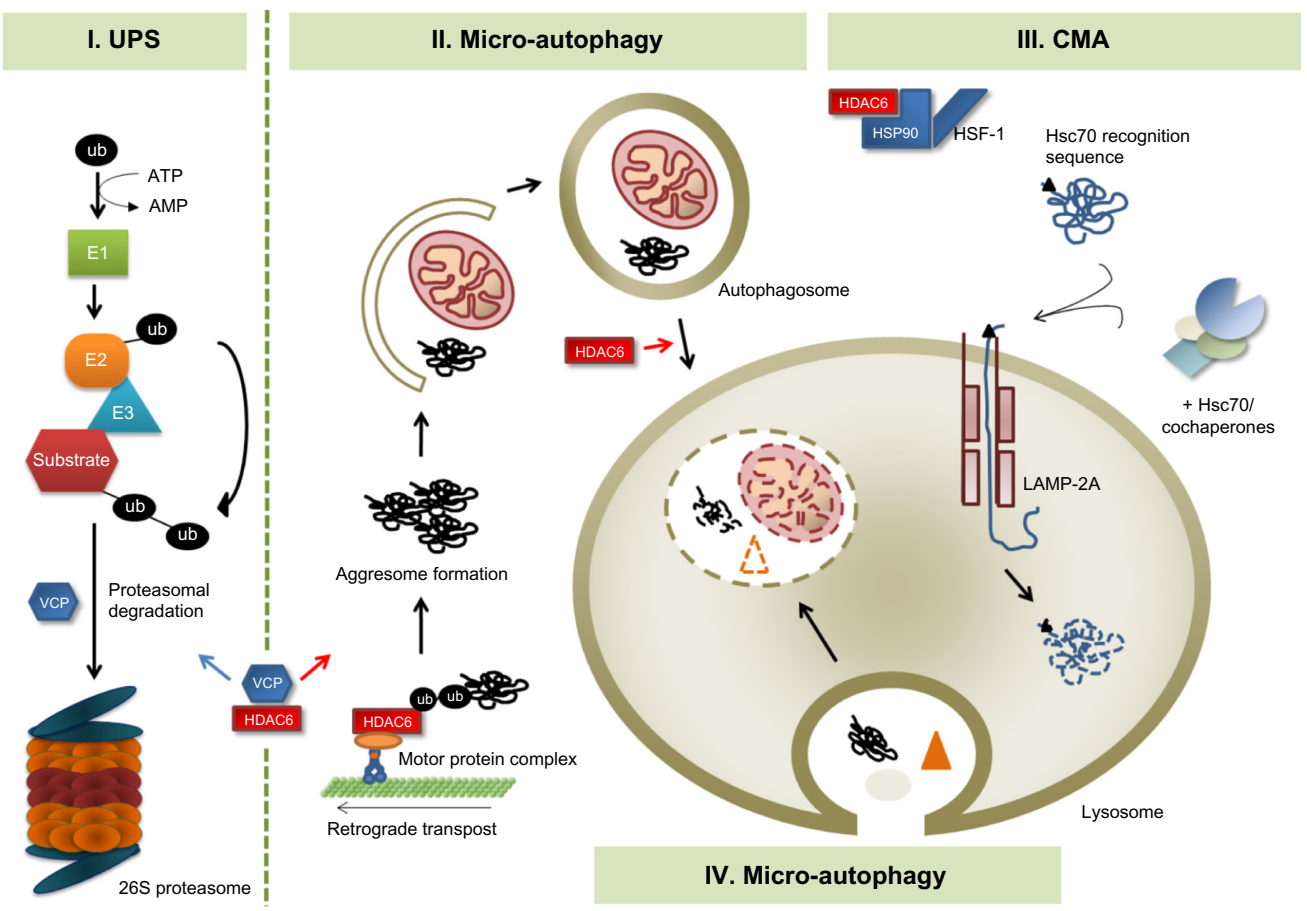

Figure I The role of HDAC6 in multiple degradation pathways of the cell.

Notes: (I) UPS: under physiological conditions, abnormal and misfolded proteins are degraded through the UPS. First, these proteins receive a poly-ubiquitin tag via a multistep pathway involving several ligases and conjugation enzymes (EI-E3). Next, VCP/p97, currently dominating in the VCP/P97-HDAC6 complex, dissociates ubiquitinated proteins from HDAC6 and activates the UPS. Substrates will then be transported to the proteasome for degradation. (II) Macro-autophagy: under pathological conditions, the UPS system can be saturated or dysfunctional. In such cases, cells rely on the aggresome and/or macro-autophagy for the degradation of cytosolic components to provide cells with necessary building blocks and reduce cellular stress. When ubiquitinated proteins accumulate, the VCP/p97-HDAC6 balance will shift in favor of HDAC6. Next, HDAC6 couples the aggregates to the motor protein complex for retrograde transport. In the perinuclear region, these accumulating proteins form the aggresome, which will be engulfed by a double membrane together with other cytosolic components (eg, mitochondria), to form an autophagosome. HDAC6 further mediates the maturation of the autophagosome and promotes their fusion with the lysosome, where the content will be degraded. (III) CMA: under stress, cells will activate a pathway called the heat shock response. HDAC6 forms a complex with the chaperone HSP90 and the transcription factor HSFI. After detecting ubiquitinated proteins, HDAC6 mediated the dissociation of the complex, resulting in the release and activation of HSFI. This will promote the transcription of different heat shock proteins and co-chaperones. These factors will form a complex that can recognize proteins and delivers them to the lysosome via LAMP-2A. (IV) Micro-autophagy: the last form of autophagy is micro-autophagy. HDAC6 promotes the maturation of lysosomes, which can then engulf cytosolic components directly by invagination. In contrast to macro-autophagy, the formation of autophagosomes is not required.

Abbreviations: CMA, chaperone-mediated autophagy; UPS, ubiquitin-proteasome system.

rely on alternative pathways to deal with protein-induced stress. The cytotoxic aggregates can then be cleared by chaperone-mediated autophagy or by macro-autophagy.

\section{Autophagy}

Degradation of proteins and organelles inside cellular inclusions was first described by Christian de Duve in $1963 .{ }^{50}$ This catabolic process is used for the recycling of cellular components to generate macromolecular building blocks and includes three different types: unselective micro-autophagy, macro-autophagy, and chaperone-mediated autophagy (Figure 1). In micro-autophagy, cytosolic components are directly engulfed by invagination of the lysosomal membrane for degradation. During macro-autophagy, also referred to as the endoplasmic reticulum-associated degradation pathway, the cytosolic substrates are surrounded by a double membrane, which initiates primarily from the endoplasmic reticulum, forming the autophagosome. In later stages, this structure fuses with lysosomes and subsequently the substrates are degraded. ${ }^{51}$ Unlike conventional micro- and macro-autophagy, chaperone-mediated autophagy does not require the formation of autophagosomes. Instead, the substrates contain a consensus sequence that is recognized by Hsc70, after which Hsc70 and its co-chaperones form a complex and interact with LAMP-2A. This interaction is followed by direct translocation of the substrates across the membrane to the lysosomal lumen for subsequent degradation. ${ }^{52}$ Interestingly, HDAC6 has been reported to play a role at different levels in these processes that cope with cytotoxic aggregates. ${ }^{43}$

First of all and due to the high-affinity binding of HDAC6 to ubiquitin, it was suggested that chaperones are involved in the regulation of the ubiquitin-dependent functions of HDAC6. ${ }^{53} \mathrm{~A}$ beacon molecule in the degradation pathway is the chaperone-like $97 \mathrm{kDa} \mathrm{VCP} / \mathrm{p} 97$. This adenosine triphosphatase associated with diverse cellular activities plays a 
pivotal role in the targeting of poly-ubiquitinated proteins to the proteasome, a process that can be subdivided into three steps. First, substrates that require degradation have to be recognized, which is mediated by the ubiquitin conjugation system. Second, poly-ubiquitinated proteins are translocated. Finally, these proteins are degraded by the $26 \mathrm{~S}$ proteasome. ${ }^{54}$ $\mathrm{VCP} / \mathrm{p} 97$ governs critical steps in this process such as retrotranslocation and transportation of ubiquitinated proteins to the proteasome. ${ }^{55}$ The interaction between $\mathrm{VCP} / \mathrm{p} 97$ and HDAC6 is important in the decision whether ubiquitinated proteins are cleared by proteasome-mediated degradation or by macro-autophagy. Indeed, it was shown that $\mathrm{VCP} / \mathrm{p} 97$ interacts with HDAC6 and can exert its segregase activity to release HDAC6 from ubiquitinated proteins, favoring proteasomal clearance (Figure 1). ${ }^{56}$ However, when excess proteins accumulate and aggregate, the VCP/p97-HDAC6 balance will shift. This can be due to a decrease in VCP/p97 levels, an increase in HDAC6 expression, or both. It allows HDAC6 to mediate protein delivery to the dynein/dynactin motor complex for retrograde transport and induce the formation of an inclusion body, also known as the aggresome. ${ }^{57}$ These data indicate that HDAC6 does not only link protein ubiquitination to the proteasome, but also to the cytoskeletondependent degradation and autophagy. ${ }^{6}$

Second, HDAC6 interacts with HSP90 and regulates its chaperone activity as mentioned above. ${ }^{3}$ Together with HSF1 and HSP90, HDAC6 forms a complex in which HSF1 is maintained in an inactive form by HSP90. HDAC6-mediated detachment of the HSP90/HSF1 complex is required for activation of the heat shock response. It was suggested that HDAC6 forms a bridge between protein-induced stress and activation of this pathway in neurons that are continuously exposed to toxic insults during the course of disease. ${ }^{43}$ After detecting the presence of ubiquitinated proteins, HDAC6 will deacetylate HSP90, promoting dissociation of the HDAC6/ HSP90/HSF1 complex and stimulating a heat shock response. Heat shock proteins are known to associate with ubiquitinated proteins, to induce chaperone-mediated autophagy, and to prevent the formation of cytotoxic aggregates. ${ }^{58}$ In addition to its well-known role in malignancies, HSP90 is also important for maintaining the stability of neuronal proteins associated with neurodegenerative disorders, which could promote the accumulation of toxic aggregates. ${ }^{59}$ As a consequence, modulation of HDAC6 activity could be a therapeutic strategy in neurodegeneration.

Third, a study from Pandey et al suggested that autophagy compensates for proteasome deficiency in an HDAC6dependent manner. ${ }^{60}$ Although HDAC6 is not essential for autophagy activation per se, it seems to be implicated in the maturation of lysosomes and their subsequent fusion with autophagosomes. ${ }^{61}$ The molecular determinants for the formation of autophagic vacuoles and maturation of these autophagosomes are called Atg proteins. ${ }^{62}$ Lysosomes and Atg proteins are recruited to the perinuclear aggresome by a process that requires HDAC6. ${ }^{61}$ In fact, loss of HDAC6 impairs the recruitment of lysosomes and LC3, a well established indicator for autophagic processing, to the aggresome. ${ }^{63}$ Furthermore, the F-actin cytoskeleton is also essential in autophagosome-lysosomal fusion. ${ }^{64} \mathrm{~F}$-actin remodeling is regulated through reversible deacetylation of cortactin and is thus controlled by HDAC6. ${ }^{4}$

In conclusion, HDAC6 is required for 1) retrograde transport of ubiquitinated aggregates - functioning as a bridge between ubiquitin and the dynein/dynactin motor complex, 2) formation of the aggresome, and 3) fusion of autophagosomes with lysosomes through modulation of the F-actin cytoskeleton. ${ }^{43,63-65}$

\section{Stress granules}

HDAC6 has been associated with stress granules - cytoplasmic structures in which a reversible block of messenger RNA (mRNA) translation occurs after cellular stress. ${ }^{66}$ Dysfunction of the proteasome does not only induce autophagy but also an increased formation of stress granules. ${ }^{67}$ Intriguingly, HDAC6 co-localizes with stress granules and interacts with G3BP - another stress granule protein. ${ }^{68}$ Phosphorylation of G3BP reduces the interaction with HDAC6 and decreases the formation of stress granules. Furthermore, disruption of the microtubules or the dynein/dynactin motor complex, as well as pharmacological or genetic inhibition of HDAC6, abolishes the formation of stress granules. ${ }^{68}$ These results indicate a modulatory role for HDAC6 in both autophagy and the formation of stress granules after cellular stress.

\section{Conclusions on HDAC6 mechanisms}

In summary, HDAC6 functions as an important regulator in a variety of biological pathways in dividing cells, but also in post-mitotic neurons. In these cells, survival is more dependent on HDAC6-mediated processes such as intracellular trafficking, antioxidation, chaperone-mediated stress responses, and protein degradation..$^{7,28,43,65}$ Over the last decade, interest in the role of HDAC6 in disease has grown significantly, especially its role in cancer (reviewed in Aldana-Masangkay and Sakamoto ${ }^{69}$ ) and in neurodegeneration (discussed in the next part of this review in which we highlight the key role of HDAC6 in neurodegeneration). In neurons, it was suggested 
that HDAC6 plays a dual role as it balances between neuroprotection and neurodegeneration. ${ }^{9}$ HDAC6-dependent deacetylation of its substrates can shift the balance towards neurotoxic insults, whereas the ubiquitin-dependent activities of HDAC6 promote neuroprotection. As a consequence, the use of compounds that specifically inhibit the deacetylating activity of HDAC6, while leaving its functions in stress response and protein degradation unaltered, might be the treatment of choice for several diseases, including neurodegenerative disorders. Several HDAC6-specific inhibitors with improved brain permeability are being developed, enhancing their therapeutic potential in neurodegeneration..$^{70}$ To date, only one HDAC6 inhibitor (ACY-1215) is being tested in a clinical trial for multiple myeloma. ${ }^{71}$ The bioavailability in the central nervous system has not been studied in humans so far, but it would be interesting to evaluate its potential for clinical use in neurological disorders.

\section{HDAC6 as a key player in neurodegeneration}

AD, PD, HD, ALS, spinal muscular atrophy (SMA), and Charcot-Marie-Tooth disease (CMT) are well known examples of neurodegenerative disorders. These diseases have some pathophysiological hallmarks in common. These include amongst others: dysfunctional mitochondria, reduced axonal transport, abnormal aggregation of misfolded proteins, and increased oxidative stress. ${ }^{72-75}$ Intriguingly, HDAC6 fulfills a regulatory role in many of these processes as discussed in the first part of this review on the molecular functions of HDAC6. This part of the review summarizes the available information on HDAC6 involvement in different neurodegenerative disorders (Table 1).

\section{AD}

$\mathrm{AD}$ is the most common form of dementia characterized by the deposition of amyloid- $\beta_{42}$ plaques and neurofibrillary tau tangles. This leads to neuronal loss in specific areas of the brain. Abnormal tau phosphorylation and subsequent accumulation of neurofibrillary tau tangles was proposed to compromise microtubule dynamics and neuronal function. ${ }^{76}$ As HDAC6 directly binds to tau, both in vitro and in vivo, and influences the phosphorylation and incorporation of tau into neurofibrillary tau tangles, it is a potential modifier of AD pathology. ${ }^{77,78}$ A positive correlation between HDAC6 and tau burden was reported as a loss of HDAC6 function or expression attenuates the phosphorylation of tau and promotes its clearance. ${ }^{77,79}$ This was confirmed in a mouse model for AD treated with two different HDAC6 inhibitors. ${ }^{80}$ More recently, it was confirmed that HDAC6 inhibition or deficiency modulates tau acetylation, phosphorylation, and isoform expression in oligodendrocytes, ${ }^{81}$ emphasizing the link between HDAC6 and AD. Furthermore, HDAC6 expression is upregulated in hippocampal neurons exposed to amyloid- $\beta$ plaques in vitro, ${ }^{82}$ in the brain of a mouse model for $\mathrm{AD},{ }^{82}$ and in the hippocampus of $\mathrm{AD}$ patients. ${ }^{77,83}$ A recent study found similar HDAC6 levels in the temporal neocortex of $\mathrm{AD}$ patients and controls,${ }^{84}$ indicating that the increase in HDAC6 expression is limited to hippocampal neurons, especially those with a high tau burden. These studies suggest an HDAC6-mediated compensatory response to stress. Unexpectedly, another study found an increase in the acetylation level of $\alpha$-tubulin in the same brain region of AD patients in combination with an upregulation of HDAC6 expression. ${ }^{83,85}$ This discrepancy might be explained by the fact that tau directly inhibits HDAC6 function. ${ }^{83}$ It could be that neurons activate a compensatory increase in HDAC6 expression to deal with the protein stress following the accumulation of amyloid- $\beta$ and tau. However, the excess tau can directly inhibit the function of the HDAC6 protein resulting in increased $\alpha$-tubulin acetylation. ${ }^{77,83}$

Despite these conflicting results, the potential use of HDAC6 inhibitors as a treatment for $\mathrm{AD}$ was emphasized by two independent studies in which it was shown that specific HDAC6 inhibition can rescue the deficits in mitochondrial transport induced by amyloid- $\beta$ toxicity in hippocampal neurons. ${ }^{28,82}$ Similarly, inhibition of the deacetylase activity of HDAC6 restores tau-induced microtubule defects in Drosophila ${ }^{86}$ and cognitive defects in $\mathrm{AD}$ mice ${ }^{87}$ The finding that reducing endogenous HDAC6 ameliorates cognitive deficits in a mouse model for $\mathrm{AD}$ by restoring $\alpha$-tubulin acetylation and mitochondrial trafficking $^{88}$ further strengthens the potential of HDAC6 as a suitable therapeutic target in AD.

Taken together, these studies imply both a protective and a detrimental role of HDAC6 in the pathophysiology of AD. Although this may seem counterintuitive, the apparent paradox could be explained by the dual function of HDAC6 in AD. On one hand, HDAC6 is recruited to accumulating aggregates. Next, HDAC6 binds these ubiquitinated aggregates and couples them to the dynein/dynactin motor complex to promote their transport and inclusion in the aggresome, followed by the degradation of these cytotoxic aggregates. This ubiquitin-dependent function of HDAC6 could be beneficial and promote neuronal survival. On the other hand, HDAC6 can increase neuronal stress by deacetylating tau. 
Table I The role of HDAC6 in different neurodegenerative diseases

\begin{tabular}{|c|c|c|c|}
\hline Disease & HDAC6 in patients & Role of HDAC6 & References \\
\hline \multirow[t]{6}{*}{ Alzheimer's disease } & Increased in & Positive & \\
\hline & hippocampus ${ }^{77,83}$ & - Reduces protein stress induced by amyloid- $\beta$ and NFTs via the aggresome & $77-79,81,86$ \\
\hline & & Negative & \\
\hline & & - Promotes phosphorylation of tau and formation of NFTs & $77-80$ \\
\hline & & - Disturbs axonal transport of mitochondria in mice and Drosophila & $28,82,86,88$ \\
\hline & & - Inhibition of HDAC6 restores cognitive defects in Alzheimer disease mice & $80,87,88$ \\
\hline \multirow[t]{4}{*}{ Parkinson's disease } & Unknown & Positive & \\
\hline & & $\begin{array}{l}\text { - Reduces protein stress induced by } \alpha \text {-synuclein via Lewy bodies and aggresome } \\
\text { formation }\end{array}$ & $90-92$ \\
\hline & & - Protects dopaminergic neurons in Drosophila & 91 \\
\hline & & - Promotes clearance of dysfunctional mitochondria & 93 \\
\hline \multirow[t]{4}{*}{ Huntington's disease } & Unknown & Positive & \\
\hline & Note: reduced & - Reduces protein stress by aggregated $\mathrm{Htt}$ & 63 \\
\hline & $\alpha$-tubulin acetylation & Negative & \\
\hline & in striatum ${ }^{30}$ & - Disturbs axonal transport of BDNF & 30 \\
\hline Amyotrophic lateral & Unknown & Positive & \\
\hline \multirow[t]{5}{*}{ sclerosis } & Note: increased GFAP & - Reduces protein stress by mutant SODI & 104 \\
\hline & acetylation in spinal & - Promotes stress granule formation and neurite outgrowth & 68,98 \\
\hline & cord astrocytes $^{108}$ & Negative & \\
\hline & & - Genetic deletion delays disease progression in mutant SODI mice & 102 \\
\hline & & - HDAC inhibition protects against motor neuron death and axonal degeneration & 103 \\
\hline Spinal muscular & Unknown & Positive & \\
\hline \multirow[t]{4}{*}{ atrophy } & & $\begin{array}{l}\text { - Promotes expression of full-length SMN2 by sequestering aberrantly spliced } \\
\text { SMN in the aggresome }\end{array}$ & 112 \\
\hline & & - Ameliorates neurodegenerative phenotype in Drosophila & 60 \\
\hline & & Negative & \\
\hline & & - Reduces axonal transport in sciatic nerve of SMN $\Delta 7$ mice & 114 \\
\hline Charcot-Marie- & Unknown & Positive & \\
\hline \multirow[t]{4}{*}{ Tooth disease } & & - Reduces protein stress by induction of aggresome-autophagy pathway & $119,121,122$ \\
\hline & & Negative & \\
\hline & & - Blocks axonal transport of mitochondria via deacetylating $\alpha$-tubulin & 123 \\
\hline & & - Pharmacological inhibition rescues phenotype in mutant HSPBI mice & 123 \\
\hline \multirow[t]{8}{*}{ Common hallmarks } & & Positive - ubiquitin-dependent functions & \\
\hline & & - Promotes protein degradation via the aggresome-autophagy pathways & \\
\hline & & - Promotes chaperone-mediated stress response & \\
\hline & & - Promotes formation of stress granules & \\
\hline & & Negative - deacetylase-dependent functions & \\
\hline & & - Disturbs axonal transport by deacetylating $\alpha$-tubulin & \\
\hline & & - Disturbs actin cytoskeleton by deacetylating cortactin & \\
\hline & & - Reduces antioxidant capacity by deacetylating peroxiredoxins & \\
\hline
\end{tabular}

Abbreviations: BDNF, brain-derived neurotrophic factor; NFT, neurofibrillary tangle; SODI, superoxide dismutase I.

Acetylation and phosphorylation of tau occurs on the same motif and HDAC6-mediated deacetylation of tau renders this motif available for phosphorylation. ${ }^{78}$ Whereas acetylation would prevent the aggregation and increase the clearance of tau, phosphorylation promotes the formation of neurofibrillary tau tangles. In accordance with this, it was reported that these motifs are hypoacetylated in $\mathrm{AD}$ patients, leading to increased neuronal stress. ${ }^{78}$ As a consequence, inhibiting the deacetylase activity of HDAC6, while leaving its role in protein degradation unhampered, could be an interesting strategy to treat $\mathrm{AD}$.

\section{PD}

$\mathrm{PD}$ is the second most common neurodegenerative disease, caused by a selective loss of dopaminergic neurons in the substantia nigra. Although neuronal death is mainly idiopathic, aberrant protein aggregation is most likely the key factor in the development of PD as neuronal lesions are accompanied by aggresome-like inclusion bodies, called Lewy bodies. ${ }^{89}$ Furthermore, Lewy bodies sequester ubiquitin-activating enzyme E1 and Hsp70, two enzymes that are recruited to the aggresome in the HDAC6-mediated response to protein stress. ${ }^{6}$ The importance of HDAC6 in PD 
is emphasized by a study demonstrating the co-localization of HDAC6 with $\alpha$-synuclein - a genetic cause of familial PD - inside the Lewy bodies in brain sections of PD patients. ${ }^{44}$ Recently, it was suggested that HDAC6-mediated protein degradation is protective in PD and dementia with Lewy bodies. ${ }^{90}$ In this study, overexpression of mutant $\alpha$-synuclein or 1-methyl-4-phenylpyridinium-induced neuronal stress significantly increases HDAC6 expression in PC-12 cells. ${ }^{90}$ Likewise, HDAC6 inhibition reduces the autophagic response and the formation of Lewy bodies, followed by an impaired clearance of mutant $\alpha$-synuclein. ${ }^{90}$ The protective nature of HDAC6 was confirmed by a study in Drosophila in which the endogenous Hdac6 protects dopaminergic neurons against cytotoxic $\alpha$-synuclein aggregates by stimulating aggresome-like inclusion formation. ${ }^{91}$ Moreover, the E3 ligase parkin, mutated in autosomal recessive PD, mediates the polyubiquitination of DJ-1, which serves as a signal for HDAC6 to promote the sequestration of ubiquitinated aggregates into the aggresome. ${ }^{92}$ Parkin also mediates HDAC6-dependent mitophagy. After ubiquitination of dysfunctional mitochondria by parkin, the autophagic mediators HDAC6 and p62 are recruited and mitochondrial clearance is enhanced..$^{93}$ Taken together, these results are in favor of a neuroprotective role of HDAC6 and for the potential use of HDAC6 agonists as a therapeutic strategy in PD. So far, no HDAC6 agonists have been studied.

\section{HD}

$\mathrm{HD}$ is a neurodegenerative disease elicited by an abnormal polyglutamine expansion in the huntingtin protein. HD patients display defects in motor coordination (chorea) and cognitive function. It was suggested that polyglutamine proteins cause neuronal dysfunction in Drosophila by two mechanisms: accumulation of aggregated huntingtin and disturbances in axonal transport. ${ }^{94}$ Although it was originally thought that these mechanisms were independent, HDAC6 is now associated with both processes. ${ }^{6,27}$ Indeed, HDAC6-dependent retrograde transport mediates clearance of aggregated huntingtin in neuronal cells, ${ }^{63}$ while HDAC6 inhibition increases $\alpha$-tubulin acetylation and restores axonal transport of vesicles containing BDNF. ${ }^{30}$ Moreover, brains of HD patients show a significant reduction in acetylated $\alpha$-tubulin..$^{30}$ These data suggest that HDAC6 could be a good therapeutic target. However, the use of HDAC6 inhibitors to treat HD is still under discussion. Bobrowska et al showed that HDAC6 knockout is not able to modify disease progression in a mouse model of HD, although $\alpha$-tubulin acetylation increases. ${ }^{95}$ Another study observed improvements of the neurodegenerative phenotype in the same mouse model after inhibition of SIRT2, another tubulin deacetylating enzyme belonging to the class III HDACs. ${ }^{96}$ As a consequence, the potential of HDAC6 as a therapeutic target to treat HD needs to be further investigated.

\section{ALS}

ALS is a fatal neurodegenerative motor neuron disease characterized by the selective loss of motor neurons in the spinal cord, brainstem, and motor cortex. This results in progressive muscle atrophy and paralysis. Most patients decease within 5 years after diagnosis due to respiratory failure. Familial cases of ALS have been associated with mutations in Tar DNA binding protein 43 (TDP-43) and fused in sarcoma/ translocated in sacroma (FUS/TLS), two RNA-binding proteins that co-localize with HDAC6 in stress granules. ${ }^{68} \mathrm{In}$ fact, TDP-43 and FUS/TLS are both essential components of a complex that regulates HDAC6 mRNA. ${ }^{97}$ TDP-43 knockdown negatively influences HDAC6 expression, which results in decreased neurite outgrowth of human neuroblastoma cells. ${ }^{98}$ The finding that ALS-causing mutations in TDP-43 impair the microtubule-dependent axonal transport of mRNAs ${ }^{99}$ further supports the assumption that HDAC6 could be involved in ALS. In addition, mutations in VCP/p97 are also associated with $\mathrm{ALS}^{100}$ and the link between $\mathrm{VCP} /$ p97 and HDAC6, both orchestrating the fate of ubiquitinated proteins, is well established. ${ }^{53}$ As ubiquitin-positive aggregates are present in several mouse models as well as in ALS patients, ${ }^{101}$ HDAC6 could be implicated in ALS pathogenesis. Some evidence was found supporting this notion as genetic deletion of Hdac 6 delays disease progression and increases survival in the mutant SOD1 ${ }^{\mathrm{G} 93 \mathrm{~A}}$ mouse model for ALS. ${ }^{102}$ In addition, treatment of symptomatic SOD $1{ }^{\mathrm{G} 93 \mathrm{~A}}$ mice with a general HDAC inhibitor, trichostatin A, protects against motor neuron death and axonal degeneration. ${ }^{103}$ Moreover, it was suggested that HDAC6 is directly involved in aggregation and turnover of mutant SOD1 in the same mouse model. ${ }^{104}$ Furthermore, mutations in ubiquilin-2 (UBQLN-2) cause familial ALS and are observed in spinal cord neuronal inclusions of ALS patients. ${ }^{105}$ UBQLN-2 functionally links the ubiquitination pathway with the ubiquitin-proteasome system to promote in vivo protein degradation, ${ }^{105}$ highlighting the importance of the autophagy pathway in ALS with a possible role for HDAC6. De Zoeten et al found that inhibition of HDAC6 improves the function of Foxp $3^{+}$regulatory T-cells, ${ }^{38}$ which could in theory reduce neuroinflammation and disease progression in ALS mice. ${ }^{106,107}$ Additionally, a proteomic analysis found differences in protein acetylation 
in the post-mortem spinal cords between ALS and non-ALS subjects, suggesting that HDAC6 activity is altered during disease. ${ }^{108}$ Altogether, these observations indicate a potential role for HDAC6 in ALS.

\section{SMA}

SMA is the most common fatal neuromuscular disease in infants and is in most cases caused by mutations in the gene encoding SMN. A nearly identical gene copy (SMN2) is still present in these patients, but is unable to protect from disease due to aberrant splicing of exon 7. Insufficient levels of the SMN protein cause $\alpha$-motor neuron death and eventually patients will suffer from fatal respiratory deficiencies. To date, no effective cure is available although activating SMN2 expression is a popular strategy in preclinical studies. ${ }^{109,110}$ It was shown that inhibition of HDACs could increase SMN protein levels in vitro and in vivo ${ }^{109}$ and could improve the survival of a mouse model for SMA. ${ }^{110}$ Valproic acid, an antiepileptic drug with HDAC inhibitory effects, is currently tested in a clinical trial in children with SMA (NCT01671384). Although the positive effects most likely result from histone modification, a potential role for HDAC6 in SMA has also been proposed. ${ }^{111}$ Sequestering aberrantly spliced SMN2 in inclusion bodies would promote expression of full-length SMN2, thus increasing functional SMN protein levels. ${ }^{112}$ As a consequence, HDAC6 might be important in the pathophysiology of SMA due to its role in the formation of the aggresome and stress granules. Furthermore, Pandey et al found that HDAC6 overexpression ameliorated the neurodegenerative phenotype in a Drosophila model for SMA. ${ }^{60}$ Although there is still some controversy on the presence of axonal transport deficits in SMA, the influence of HDAC6 on microtubule dynamics could also play a role in SMA. While one study failed to detect defects of intracellular trafficking in cultured embryonic motor neurons from a mouse model for $\mathrm{SMA},{ }^{113}$ another group discovered a reduction in fast axonal transport in the sciatic nerve of symptomatic SMA $\Delta 7$ mice. ${ }^{114}$ Nevertheless, it would be interesting to evaluate whether inhibition of the $\alpha$-tubulin deacetylating activity of HDAC6 could restore axonal transport, prevent motor neuron death, and improve the SMA phenotype.

\section{CMT}

CMT is the most common non-lethal hereditary disease of the peripheral nervous system with more than 70 associated genes identified so far. ${ }^{115}$ Clinical symptoms include progressive muscle wasting that starts distally ("stockingglove" pattern), foot and hand deformities, sensory deficits, and steppage gait. A variety of animal models were created based on the identification of CMT-causing genes. Remarkably, several of these models show a similar pathophysiology with deficits in axonal transport, ${ }^{116-118}$ aggregation of mutated proteins, ${ }^{119,120}$ and altered protein degradation by the proteasome and aggresome-autophagy pathway. ${ }^{19,121,122}$ Evidence was found for a role of HDAC6 in the pathophysiology of CMT. ${ }^{123}$ A mutant HSPB1-induced CMT mouse model was created, recapitulating the clinical features observed in patients and with a reduction in acetylated $\alpha$-tubulin. Moreover, cultured dorsal root ganglion neurons show severe defects in axonal transport. ${ }^{123}$ Pharmacological inhibition of the deacetylating activity of HDAC6 rescues the phenotype of mutant HSPB1-expressing mice, both at the cellular and the behavioral level. ${ }^{123}$ As a consequence, HDAC6 is a good therapeutic target in CMT.

\section{Conclusions}

These different studies provide strong evidence that HDACs, and in particular HDAC6, are important in a wide range of neurodegenerative disorders. Moreover, HDAC6 might be the common link through its regulatory function in the stress response, protein degradation, and axonal transport - different processes that are all altered in the abovementioned diseases. Altogether, it appears that HDAC6 has both neuroprotective and neurodegenerative properties. In neurodegenerative disorders, ubiquitinated aggregates accumulate during the course of the disease and increase the cellular burden. Subsequently, stressinduced cellular responses will target these aggregates to the proteasome for degradation. The increased protein load will eventually saturate this first line of defense and HDAC6 could be recruited to the accumulating ubiquitinated proteins. Through its interaction with $\mathrm{VCP} / \mathrm{p} 97$ and ubiquitin, HDAC6 favors protein degradation by alternative pathways such as aggresome formation and autophagy. HDAC6 facilitates the delivery of ubiquitinated proteins to the aggresome by recruiting the motor protein dynein, which transports the aggregates to the perinuclear region, thus promoting their clearance. Simultaneously, HDAC6 will recruit components of the autophagic machinery and stimulate the autophagosome-lysosomal fusion. Finally, HDAC6 will contribute to the formation of stress granules to ensure the conservation of untranslated mRNAs during cellular stress. All together, these cellular effects emphasize the neuroprotective nature of HDAC6. However, as HDAC6 is recruited to ubiquitinated aggregates, it may also cause excessive deacetylation of its substrates, generating an accumulation 
of neurotoxic insults. For example, increased deacetylation of $\alpha$-tubulin or cortactin can disturb the microtubule and actin dynamics, which affect the cytoskeletal structure in neurons. Furthermore, the recruitment and anchoring of motor proteins to the microtubules is reduced following deacetylation of $\alpha$-tubulin. Moreover, HDAC6-mediated deacetylation of peroxiredoxins will increase oxidative stress, eventually contributing to neurodegeneration.

In conclusion, specific inhibition of the deacetylating function with specific inhibitors could be a good strategy to eliminate the negative aspects of HDAC6, while leaving its protective role in the stress response and protein degradation unhampered. As a consequence, the authors believe that this could be a good therapeutic approach in different forms of neurodegeneration.

\section{Acknowledgments}

Research of the authors is supported by grants from the VIB (Institute for Biotechnology Flanders), the University of Leuven, the Belgian government (Interuniversity Attraction Poles of the Belgian Federal Science Policy Office), the Muscular Dystrophy Association (MDA), the ALS Liga Belgium, the ALS Therapy Alliance, the Association Belge contre les Maladies neuro-Musculaires (ABMM), and the National Institute of Health (NIH). LVH and VB are supported by the Agency for Innovation by Science and Technologies (IWT).

\section{Disclosure}

The authors report no conflicts of interest in this work.

\section{References}

1. Li G, Jiang H, Chang M, Xie H, Hu L. HDAC6 $\alpha$-tubulin deacetylase: a potential therapeutic target in neurodegenerative diseases. $J$ Neurol Sci. 2011;304(1-2):1-8.

2. Zhang Y, Li N, Caron C, et al. HDAC-6 interacts with and deacetylates tubulin and microtubules in vivo. EMBO J. 2003;22(5):1168-1179.

3. Kovacs JJ, Murphy PJ, Gaillard S, et al. HDAC6 regulates Hsp90 acetylation and chaperone-dependent activation of glucocorticoid receptor. Mol Cell. 2005;18(5):601-607.

4. Zhang X, Yuan Z, Zhang Y, et al. HDAC6 modulates cell motility by altering the acetylation level of cortactin. Mol Cell. 2007;27(2):197-213.

5. Ding G, Liu HD, Huang Q, et al. HDAC6 promotes hepatocellular carcinoma progression by inhibiting P53 transcriptional activity. FEBS Lett. 2013;587(7):880-886.

6. Kawaguchi Y, Kovacs JJ, McLaurin A, Vance JM, Ito A, Yao TP. The deacetylase HDAC6 regulates aggresome formation and cell viability in response to misfolded protein stress. Cell. 2003;115(6):727-738.

7. Parmigiani RB, Xu WS, Venta-Perez G, et al. HDAC6 is a specific deacetylase of peroxiredoxins and is involved in redox regulation. Proc Natl Acad Sci U S A. 2008;105(28):9633-9638.

8. Grunstein M. Histone acetylation in chromatin structure and transcription. Nature. 1997;389(6649):349-352.
9. d'Ydewalle C, Bogaert E, Van Den Bosch L. HDAC6 at the intersection of neuroprotection and neurodegeneration. Traffic. 2012;13(6): 771-779.

10. de Ruijter AJ, van Gennip AH, Caron HN, Kemp S, van Kuilenburg AB. Histone deacetylases (HDACs): characterization of the classical HDAC family. Biochem J. 2003;370(Pt 3):737-749.

11. Imai S, Armstrong CM, Kaeberlein M, Guarente L. Transcriptional silencing and longevity protein Sir2 is an NAD-dependent histone deacetylase. Nature. 2000;403(6771):795-800.

12. Polo SE, Almouzni G. Histone metabolic pathways and chromatin assembly factors as proliferation markers. Cancer Lett. 2005;220(1):1-9.

13. Kim HJ, Bae SC. Histone deacetylase inhibitors: molecular mechanisms of action and clinical trials as anti-cancer drugs. Am J Transl Res. 2011;3(2):166-179.

14. Prince HM, Bishton MJ, Harrison SJ. Clinical studies of histone deacetylase inhibitors. Clin Cancer Res. 2009;15(12):3958-3969.

15. Thaler F, Mercurio C. Towards selective inhibition of histone deacetylase isoforms: what has been achieved, where we are, and what will be next. ChemMedChem. 2014;9(3):523-526.

16. Bertos NR, Gilquin B, Chan GK, Yen TJ, Khochbin S, Yang XJ. Role of the tetradecapeptide repeat domain of human histone deacetylase 6 in cytoplasmic retention. J Biol Chem. 2004;279(46):48246-48254.

17. Westermann S, Weber K. Post-translational modifications regulate microtubule function. Nat Rev Mol Cell Biol. 2003;4(12):938-947.

18. Maruta H, Greer K, Rosenbaum JL. The acetylation of $\alpha$-tubulin and its relationship to the assembly and disassembly of microtubules. $J$ Cell Biol. 1986;103(2):571-579.

19. Janke C, Bulinski JC. Post-translational regulation of the microtubule cytoskeleton: mechanisms and functions. Nat Rev Mol Cell Biol. 2011;12(12):773-786.

20. Hubbert C, Guardiola A, Shao R, et al. HDAC6 is a microtubuleassociated deacetylase. Nature. 2002;417(6887):455-458.

21. Matsuyama A, Shimazu T, Sumida Y, et al. In vivo destabilization of dynamic microtubules by HDAC6-mediated deacetylation. EMBO J. 2002;21(24):6820-6831.

22. Zilberman Y, Ballestrem C, Carramusa L, Mazitschek R, Khochbin S, Bershadsky A. Regulation of microtubule dynamics by inhibition of the tubulin deacetylase HDAC6. J Cell Sci. 2009;122(Pt 19):3531-3541.

23. Brinkley BR. Microtubule organizing centers. Annu Rev Cell Biol. 1985;1:145-172.

24. Conde C, Caceres A. Microtubule assembly, organization, and dynamics in axons and dendrites. Nat Rev Neurosci. 2009;10(5):319-332.

25. Karki S, Holzbaur EL. Cytoplasmic dynein and dynactin in cell division and intracellular transport. Curr Opin Cell Biol. 1999;11(1):45-53.

26. Hirokawa N. Kinesin and dynein superfamily proteins and the mechanism of organelle transport. Science. 1998;279(5350):519-526.

27. Reed NA, Cai D, Blasius TL, et al. Microtubule acetylation promotes kinesin-1 binding and transport. Curr Biol. 2006;16(21):2166-2172.

28. Chen S, Owens GC, Makarenkova H, Edelman DB. HDAC6 regulates mitochondrial transport in hippocampal neurons. PLoS One. 2010;5(5):e10848.

29. Kopito RR. The missing linker: an unexpected role for a histone deacetylase. Mol Cell. 2003;12(6):1349-1351.

30. Dompierre JP, Godin JD, Charrin BC, et al. Histone deacetylase 6 inhibition compensates for the transport deficit in Huntington's disease by increasing tubulin acetylation. J Neurosci. 2007;27(13):3571-3583.

31. Chen S, Owens GC, Crossin KL, Edelman DB. Serotonin stimulates mitochondrial transport in hippocampal neurons. Mol Cell Neurosci. 2007;36(4):472-483.

32. Wood ZA, Schroder E, Robin Harris J, Poole LB. Structure, mechanism, and regulation of peroxiredoxins. Trends Biochem Sci. 2003;28(1): $32-40$.

33. Moon JC, Kim GM, Kim EK, et al. Reversal of 2-Cys peroxiredoxin oligomerization by sulfiredoxin. Biochem Biophys Res Commun. 2013;432(2):291-295.

34. Amor S, Puentes F, Baker D, van der Valk P. Inflammation in neurodegenerative diseases. Immunology. 2010;129(2):154-169. 
35. Amor S, Woodroofe MN. Innate and adaptive immune responses in neurodegeneration and repair. Immunology. 2014;141(3): 287-291.

36. Fontenot JD, Gavin MA, Rudensky AY. Foxp3 programs the development and function of $\mathrm{CD} 4{ }^{+} \mathrm{CD} 25^{+}$regulatory T-cells. Nat Immunol. 2003;4(4):330-336.

37. Shakespear MR, Halili MA, Irvine KM, Fairlie DP, Sweet MJ. Histone deacetylases as regulators of inflammation and immunity. Trends Immunol. 2011;32(7):335-343.

38. De Zoeten EF, Wang L, Butler K, et al. Histone deacetylase 6 and heat shock protein 90 control the functions of Foxp $3^{+}$T-regulatory cells. $\mathrm{Mol}$ Cell Biol. 2011;31(10):2066-2078.

39. Liesz A, Zhou W, Na SY, et al. Boosting regulatory T-cells limits neuroinflammation in permanent cortical stroke. J Neurosci. 2013;33(44): $17350-17362$.

40. Kleinewietfeld M, Hafler DA. Regulatory T-cells in autoimmune neuroinflammation. Immunol Rev. 2014;259(1):231-244.

41. Buchner J. Hsp90 and co. - a holding for folding. Trends Biochem Sci. 1999;24(4):136-141

42. Ali A, Bharadwaj S, O’Carroll R, Ovsenek N. HSP90 interacts with and regulates the activity of heat shock factor 1 in Xenopus oocytes. Mol Cell Biol. 1998;18(9):4949-4960.

43. Boyault $\mathrm{C}$, Zhang $\mathrm{Y}$, Fritah $\mathrm{S}$, et al. HDAC6 controls major cell response pathways to cytotoxic accumulation of protein aggregates. Genes Dev 2007;21(17):2172-2181.

44. Li Y, Zhang T, Schwartz SJ, Sun D. New developments in Hsp90 inhibitors as anti-cancer therapeutics: mechanisms, clinical perspective, and more potential. Drug Resist Updat. 2009; 12(1-2):17-27.

45. Li D, Marchenko ND, Moll UM. SAHA shows preferential cytotoxicity in mutant p53 cancer cells by destabilizing mutant p53 through inhibition of the HDAC6-Hsp90 chaperone axis. Cell Death Differ. 2011;18(12):1904-1913.

46. Gravina GL, Marampon F, Muzi P, et al. PXD101 potentiates hormonal therapy and prevents the onset of castration-resistant phenotype modulating androgen receptor, HSP90, and CRM1 in preclinical models of prostate cancer. Endocr Relat Cancer. 2013;20(3):321-337.

47. Li D, Sun X, Zhang L, et al. Histone deacetylase 6 and cytoplasmic linker protein 170 function together to regulate the motility of pancreatic cancer cells. Protein Cell. 2014;5(3):214-223.

48. Goldberg AL. Protein degradation and protection against misfolded or damaged proteins. Nature. 2003;426(6968):895-899.

49. Stoppini M, Andreola A, Foresti G, Bellotti V. Neurodegenerative diseases caused by protein aggregation: a phenomenon at the borderline between molecular evolution and ageing. Pharmacol Res. 2004;50(4): 419-431.

50. De Duve C. The lysosome. Sci Am. 1963;208:64-72.

51. Levine B, Klionsky DJ. Development by self-digestion: molecular mechanisms and biological functions of autophagy. Dev Cell. 2004;6(4) 463-477

52. Massey AC, Zhang C, Cuervo AM. Chaperone-mediated autophagy in aging and disease. Curr Top Dev Biol. 2006;73:205-235.

53. Boyault C, Gilquin B, Zhang Y, et al. HDAC6-p97/VCP controlled polyubiquitin chain turnover. EMBO J. 2006;25(14):3357-3366.

54. Bagola K, Mehnert M, Jarosch E, Sommer T. Protein dislocation from the ER. Biochim Biophys Acta. 2011;1808(3):925-936.

55. Wolf DH, Stolz A. The Cdc48 machine in endoplasmic reticulum associated protein degradation. Biochim Biophys Acta. 2012;1823(1): 117-124.

56. Seigneurin-Berny D, Verdel A, Curtet S, et al. Identification of components of the murine histone deacetylase 6 complex: link between acetylation and ubiquitination signaling pathways. Mol Cell Biol. 2001;21(23):8035-8044

57. Kopito RR. Aggresomes, inclusion bodies, and protein aggregation. Trends Cell Biol. 2000;10(12):524-530.

58. Zeng $\mathrm{XC}$, Bhasin $\mathrm{S}, \mathrm{Wu} \mathrm{X}$, et al. Hsp70 dynamics in vivo: effect of heat shock and protein aggregation. J Cell Sci. 2004;117(Pt 21): 4991-5000.
59. Waza M, Adachi H, Katsuno M, et al. Modulation of Hsp90 function in neurodegenerative disorders: a molecular-targeted therapy against disease-causing protein. J Mol Med (Berl). 2006;84(8):635-646.

60. Pandey UB, Nie Z, Batlevi Y, et al. HDAC6 rescues neurodegeneration and provides an essential link between autophagy and the UPS. Nature. 2007;447(7146):859-863.

61. Lee JY, Koga H, Kawaguchi Y, et al. HDAC6 controls autophagosome maturation essential for ubiquitin-selective quality-control autophagy. EMBO J. 2010;29(5):969-980.

62. Mizushima N, Ohsumi Y, Yoshimori T. Autophagosome formation in mammalian cells. Cell Struct Funct. 2002;27(6):421-429.

63. Iwata A, Riley BE, Johnston JA, Kopito RR. HDAC6 and microtubules are required for autophagic degradation of aggregated huntingtin. J Biol Chem. 2005;280(48):40282-40292.

64. Liebl D, Griffiths G. Transient assembly of F-actin by phagosomes delays phagosome fusion with lysosomes in cargo-overloaded macrophages. J Cell Sci. 2009;122(Pt 16):2935-2945.

65. Boyault C, Sadoul K, Pabion M, Khochbin S. HDAC6, at the crossroads between cytoskeleton and cell signaling by acetylation and ubiquitination. Oncogene. 2007;26(37):5468-5476.

66. Anderson P, Kedersha N. RNA granules. J Cell Biol. 2006;172(6): 803-808.

67. Mazroui R, Di Marco S, Kaufman RJ, Gallouzi IE. Inhibition of the ubiquitin-proteasome system induces stress granule formation. Mol Biol Cell. 2007;18(7):2603-2618.

68. Kwon S, Zhang Y, Matthias P. The deacetylase HDAC6 is a novel critical component of stress granules involved in the stress response. Genes Dev. 2007;21(24):3381-3394.

69. Aldana-Masangkay GI, Sakamoto KM. The role of HDAC6 in cancer. J Biomed Biotechnol. 2011;2011:875824.

70. Jochems J, Boulden J, Lee BG, et al. Antidepressant-like properties of novel HDAC6-selective inhibitors with improved brain bioavailability. Neuropsychopharmacology. 2014;39(2):389-400.

71. Santo L, Hideshima T, Kung AL, et al. Preclinical activity, pharmacodynamic, and pharmacokinetic properties of a selective HDAC6 inhibitor, ACY-1215, in combination with bortezomib in multiple myeloma. Blood. 2012;119(11):2579-2589.

72. Lin MT, Beal MF. Mitochondrial dysfunction and oxidative stress in neurodegenerative diseases. Nature. 2006;443(7113):787-795.

73. De Vos KJ, Grierson AJ, Ackerley S, Miller CC. Role of axonal transport in neurodegenerative diseases. Annu Rev Neurosci. 2008;31: $151-173$.

74. Ross CA, Poirier MA. Protein aggregation and neurodegenerative disease. Nat Med. 2004;10 Suppl:S10-S17.

75. Taylor JP, Hardy J, Fischbeck KH. Toxic proteins in neurodegenerative disease. Science. 2002;296(5575):1991-1995.

76. Terwel D, Dewachter I, Van Leuven F. Axonal transport, tau protein, and neurodegeneration in Alzheimer's disease. Neuromolecular Med. 2002;2(2):151-165.

77. Ding H, Dolan PJ, Johnson GV. Histone deacetylase 6 interacts with the microtubule-associated protein tau. J Neurochem. 2008;106(5): 2119-2130.

78. Cook C, Carlomagno Y, Gendron TF, et al. Acetylation of the KXGS motifs in tau is a critical determinant in modulation of tau aggregation and clearance. Hum Mol Genet. 2014;23(1):104-116.

79. Cook C, Gendron TF, Scheffel K, et al. Loss of HDAC6, a novel CHIP substrate, alleviates abnormal tau accumulation. Hum Mol Genet. 2012;21(13):2936-2945.

80. Zhang L, Liu C, Wu J, et al. Tubastatin A/ACY-1215 improves cognition in Alzheimer's disease transgenic mice. J Alzheimers Dis. Epub May 20, 2014

81. Noack M, Leyk J, Richter-Landsberg C. HDAC6 inhibition results in tau acetylation and modulates tau phosphorylation and degradation in oligodendrocytes. Glia. 2014;62(4):535-547.

82. Kim C, Choi H, Jung ES, et al. HDAC6 inhibitor blocks amyloid $\beta$-induced impairment of mitochondrial transport in hippocampal neurons. PLoS One. 2012;7(8):e42983. 
83. Perez M, Santa-Maria I, Gomez de Barreda E, et al. Tau - an inhibitor of deacetylase HDAC6 function. J Neurochem. 2009;109(6): 1756-1766.

84. Odagiri S, Tanji K, Mori F, et al. Brain expression level and activity of HDAC6 protein in neurodegenerative dementia. Biochem Biophys Res Commun. 2013;430(1):394-399.

85. Zhang L, Sheng S, Qin C. The role of HDAC6 in Alzheimer's disease. J Alzheimers Dis. 2013;33(2):283-295.

86. Cecarini V, Bonfili L, Cuccioloni M, et al. Crosstalk between the ubiquitin-proteasome system and autophagy in a human cellular model of Alzheimer's disease. Biochim Biophys Acta. 2012;1822(11): 1741-1751.

87. Selenica ML, Benner L, Housley SB, et al. Histone deacetylase 6 inhibition improves memory and reduces total tau levels in a mouse model of tau deposition. Alzheimers Res Ther. 2014;6(1):12.

88. Govindarajan N, Rao P, Burkhardt S, et al. Reducing HDAC6 ameliorates cognitive deficits in a mouse model for Alzheimer's disease. EMBO Mol Med. 2013;5(1):52-63.

89. McNaught KS, Shashidharan P, Perl DP, Jenner P, Olanow CW. Aggresome-related biogenesis of Lewy bodies. Eur J Neurosci. 2002;16(11):2136-2148.

90. Su M, Shi JJ, Yang YP, et al. HDAC6 regulates aggresome-autophagy degradation pathway of $\alpha$-synuclein in response to $\mathrm{MPP}^{+}$-induced stress. J Neurochem. 2011;117(1):112-120.

91. Du G, Liu X, Chen X, et al. Drosophila histone deacetylase 6 protects dopaminergic neurons against $\alpha$-synuclein toxicity by promoting inclusion formation. Mol Biol Cell. 2010;21(13):2128-2137.

92. Olzmann JA, Li L, Chudaev MV, et al. Parkin-mediated K63-linked polyubiquitination targets misfolded DJ-1 to aggresomes via binding to HDAC6. J Cell Biol. 2007;178(6):1025-1038.

93. Lee JY, Nagano Y, Taylor JP, Lim KL, Yao TP. Disease-causing mutations in parkin impair mitochondrial ubiquitination, aggregation, and HDAC6-dependent mitophagy. J Cell Biol. 2010;189(4):671-679.

94. Gunawardena S, Her LS, Brusch RG, et al. Disruption of axonal transport by loss of huntingtin or expression of pathogenic polyQ proteins in Drosophila. Neuron. 2003;40(1):25-40.

95. Bobrowska A, Paganetti P, Matthias P, Bates GP. Hdac6 knock-out increases tubulin acetylation but does not modify disease progression in the R6/2 mouse model of Huntington's disease. PLoS One. 2011;6(6):e20696.

96. Chopra V, Quinti L, Kim J, et al. The sirtuin 2 inhibitor AK-7 is neuroprotective in Huntington's disease mouse models. Cell Rep. 2012;2(6):1492-1497.

97. Kim SH, Shanware NP, Bowler MJ, Tibbetts RS. Amyotrophic lateral sclerosis-associated proteins TDP-43 and FUS/TLS function in a common biochemical complex to co-regulate HDAC6 mRNA. J Biol Chem. 2010;285(44):34097-34105.

98. Fiesel FC, Schurr C, Weber SS, Kahle PJ. TDP-43 knockdown impairs neurite outgrowth dependent on its target histone deacetylase $6 . \mathrm{Mol}$ Neurodegener. 2011;6:64.

99. Alami NH, Smith RB, Carrasco MA, et al. Axonal transport of TDP-43 mRNA granules is impaired by ALS-causing mutations. Neuron. 2014;81(3):536-543.

100. Johnson JO, Mandrioli J, Benatar M, et al. Exome sequencing reveals VCP mutations as a cause of familial ALS. Neuron. 2010;68(5): 857-864.

101. Blokhuis AM, Groen EJ, Koppers M, van den Berg LH, Pasterkamp RJ. Protein aggregation in amyotrophic lateral sclerosis. Acta Neuropathol. 2013;125(6):777-794.

102. Taes I, Timmers M, Hersmus N, et al. Hdac6 deletion delays disease progression in the SOD1G93A mouse model of ALS. Hum Mol Genet. 2013;22(9):1783-1790.

103. Yoo YE, Ko CP. Treatment with trichostatin A initiated after disease onset delays disease progression and increases survival in a mouse model of amyotrophic lateral sclerosis. Exp Neurol. 2011;231(1): 147-159.
104. Gal J, Chen J, Barnett KR, Yang L, Brumley E, Zhu H. HDAC6 regulates mutant SOD1 aggregation through two SMIR motifs and tubulin acetylation. J Biol Chem. 2013;288(21):15035-15045.

105. Deng HX, Chen W, Hong ST, et al. Mutations in UBQLN2 cause dominant X-linked juvenile and adult-onset ALS and ALS/dementia. Nature. 2011;477(7363):211-215.

106. Zhao W, Beers DR, Liao B, Henkel JS, Appel SH. Regulatory T-lymphocytes from ALS mice suppress microglia and effector T-lymphocytes through different cytokine-mediated mechanisms. Neurobiol Dis. 2012;48(3):418-428.

107. Henkel JS, Beers DR, Wen S, et al. Regulatory T-lymphocytes mediate amyotrophic lateral sclerosis progression and survival. EMBO Mol Med. 2013;5(1):64-79.

108. Liu D, Liu C, Li J, et al. Proteomic analysis reveals differentially regulated protein acetylation in human amyotrophic lateral sclerosis spinal cord. PLoS One. 2013;8(12):e80779.

109. Hahnen E, Eyupoglu IY, Brichta L, et al. In vitro and ex vivo evaluation of second-generation histone deacetylase inhibitors for the treatment of spinal muscular atrophy. J Neurochem. 2006; 98(1):193-202.

110. Avila AM, Burnett BG, Taye AA, et al. Trichostatin A increases SMN expression and survival in a mouse model of spinal muscular atrophy. J Clin Invest. 2007;117(3):659-671.

111. Evans MC, Cherry JJ, Androphy EJ. Differential regulation of the SMN2 gene by individual HDAC proteins. Biochem Biophys Res Commun. 2011;414(1):25-30.

112. Hofmann Y, Lorson CL, Stamm S, Androphy EJ, Wirth B. Htra2- $\beta 1$ stimulates an exonic splicing enhancer and can restore full-length SMN expression to survival motor neuron 2 (SMN2). Proc Natl Acad Sci US A. 2000;97(17):9618-9623.

113. Malik B, Nirmalananthan N, Bilsland LG, et al. Absence of disturbed axonal transport in spinal and bulbar muscular atrophy. Hum Mol Genet. 2011;20(9):1776-1786.

114. Dale JM, Shen H, Barry DM, et al. The spinal muscular atrophy mouse model, SMA $\Delta 7$, displays altered axonal transport without global neurofilament alterations. Acta Neuropathol. 2011;122(3): 331-341.

115. Department of Molecular Genetics. Inherited Peripheral Neuropathies Mutation Database (webpage on Internet). Updated 17 February 2011. Available from http://www.molgen.ua.ac.be/CMTMutations/. Accessed August 14, 2014.

116. Brownlees J, Ackerley S, Grierson AJ, et al. Charcot-Marie-Tooth disease neurofilament mutations disrupt neurofilament assembly and axonal transport. Hum Mol Genet. 2002;11(23):2837-2844.

117. Ackerley S, James PA, Kalli A, French S, Davies KE, Talbot K. A mutation in the small heat-shock protein HSPB1 leading to distal hereditary motor neuronopathy disrupts neurofilament assembly and the axonal transport of specific cellular cargoes. Hum Mol Genet. 2006;15(2):347-354.

118. Baloh RH, Schmidt RE, Pestronk A, Milbrandt J. Altered axonal mitochondrial transport in the pathogenesis of Charcot-MarieTooth disease from mitofusin 2 mutations. J Neurosci. 2007;27(2): 422-430.

119. Zhai J, Lin H, Julien JP, Schlaepfer WW. Disruption of neurofilament network with aggregation of light neurofilament protein: a common pathway leading to motor neuron degeneration due to Charcot-MarieTooth disease-linked mutations in NFL and HSPB1. Hum Mol Genet. 2007;16(24):3103-3116.

120. Lv H, Wang L, Li W, et al. Mitofusin 2 gene mutation causing earlyonset CMT2A with different progressive courses. Clin Neuropathol. 2013;32(1):16-23.

121. Lee SM, Olzmann JA, Chin LS, Li L. Mutations associated with Charcot-Marie-Tooth disease cause SIMPLE protein mislocalization and degradation by the proteasome and aggresome-autophagy pathways. J Cell Sci. 2011;124(Pt 19):3319-3331. 
122. Lee SM, Chin LS, Li L. Protein misfolding and clearance in demyelinating peripheral neuropathies: therapeutic implications. Commun Integr Biol. 2012;5(1):107-110.
123. d'Ydewalle C, Krishnan J, Chiheb DM, et al. HDAC6 inhibitors reverse axonal loss in a mouse model of mutant HSPB1-induced CharcotMarie-Tooth disease. Nat Med. 2011;17(8):968-974.

\section{Publish your work in this journal}

Research and Reports in Biology is an international, peer-reviewed, open access journal publishing original research, reports, editorials, reviews and commentaries on all areas of biology including animal biology, biochemical biology, cell biology, ecological studies, evolutionary biology, molecular biology, plant science and botany. The

\section{Dovepress}

manuscript management system is completely online and includes a very quick and fair peer-review system. Visit http://www.dovepress. $\mathrm{com} /$ testimonials.php to read real quotes from published authors. 